\title{
Novel Technique of Alcohol Impregnated Merocel Disc for Epitheliectomy Compared to Conventionally Used Alcohol Well in Photorefractive Keratectomy
}

Agarwal P*

Eye Institute, Cleveland Clinic Abu Dhabi, UAE

*Corresponding author: Prateek Agarwal, Eye Institute at Cleveland Clinic Abu Dhabi, UAE, Email: dr.prateekagarwal@gmail.com

\section{Case Report \\ Volume 5 Issue 1}

Received Date: June 16, 2020

Published Date: July 02, 2020

DOI: $10.23880 /$ oajo-16000192

\section{Abstract}

Photorefractive keratectomy has been used as a refractive modality of choice where LASIK is contraindicated. Alcohol superseded over manual scraping for epitheliectomy in photorefractive keratectomy (PRK) due to quick visual rehabilitation and less incidence of haze. The seepage of alcohol along edges of well into the adjacent conjunctiva and ocular surface causes severe postoperative pain and inflammation. It describes an innovative technique (alcohol impregnated merocel disc) for epithelial removal. The postoperative pain scoring/uncorrected visual acuity/lid edema and conjunctiva injection/size of epithelial defect/ocular surface were evaluated postoperatively. The postoperative pain and inflammatory signs (lid edema and conjunctival injection) were significantly reduced and visual acuity was better on postoperative day 1 and day 3 as compared to the control eyes in which alcohol well were used. This is a simple and cost effective technique maintaining the outcome similar to Trans epithelial PRK without being limited by the availability of specific laser platform.

Keywords: Epitheliectomy; Lasik; Keratocyte; Conjunctivae; Corneal; Mean Pain Score

Abbreviations: PRK: Photorefractive Keratectomy; LASIK: Laser in Situ Keratomileusis; UDVA: Uncorrected Distance Visual Acuity.

\section{Background}

Photorefractive keratectomy has been used as a refractive modality of choice in thin corneas, borderline topographies with the suspicion of keratoconus in patients who are not eligible candidates for laser in situ keratomileusis(LASIK) [1,2]. Manual scraping as a method for epitheliectomy in photorefractive keratectomy (PRK) was replaced by alcohol-assisted due to quicker visual rehabilitation and less incidence of haze. There is increased incidence of anterior stromal reflectivity and keratocyte density after manual scraping [3]. Alcohol epitheliectomy leaves a smooth bed with a faster healing [4].
Usually there is seepage of alcohol beneath the alcohol well into the adjacent conjunctivae and ocular surface causing postoperative pain and inflammation. This led to exploration of alternative options and transepithelial PRK was a breakthrough which has a faster healing and less postoperative discomfort compared to conventional alcohol epitheliectomy using alcohol well [5]. The limitations of transepithelial PRK is availability of SCHWIND-ESIRIS excimer laser platform [6]. We hereby describe a novel technique to prevent spillage of alcohol thereby avoiding the postoperative discomfort and pain and still maintaining the advantage of alcohol epitheliectomy without being limited by the specific laser platform.

\section{Case Presentation}

8 eyes of 4 patients were recruited. The experimental 


\section{Open Access Journal of Ophthalmology}

nature of the procedure was explained to all the patients who signed informed consent form before the study and ethical clearance was obtained. The refractive error preoperatively was in the range of -1.5 dioptres to -4.0 Dioptres and stable for the last 2 years. The patient's cleared all the Prerequisite conditions for corneal refractive surgeries. All these 4 patients had thin cornea and borderline topography and photorefractive keratectomy was planned for them. In all the right eyes alcohol impregnated Merocel disc was used for epithelial removal whereas conventional alcohol well was used in all the left eyes as a control.

\section{Investigations}

In all eyes uncorrected visual acuity improved to 20/20 with glasses corneal topography was performed which revealed inferior superior asymmetry of 1.3 diopters on anterior float in all these patients. In all 8 eyes on best fit toric posterior elevation map aspheric asymmetry index was more than 23. There were no other signs of keratoconus. Pachymetry was less than 500 in all these eyes. Cycloplegic refraction correlated well with the manifest refraction. Florescence staining revealed normal tear film with no signs of dry eyes.

\section{Differential Diagnosis}

None

\section{Treatment}

\section{Surgical Technique}

The surgeries were performed by single surgeon PA using Wave Light Excimer laser. The treatment was aimed at emmetropia. Preoperatively $0.4 \%$ oxybuprocaine hydrochloride eye drops were instilled 3 times at an interval of 3 minutes in between each cycle. After proper cleaning of lids and lashes with $10 \%$ povidone-iodine and sterile draping eyelids were opened using a lid speculum. An $8.5 \mathrm{~mm}$ Merocel disc was used commercially available as Merocel corneal light shield made of Merocel polyvinyl acetal material. Alternatively it could be prepared manually from the Visiwipe instrument wipe (beaver visitec international inc.) made of merocel polyvinyl acetal material marking an outline with alcohol well and using scissors to cut it out.

The circular disc was impregnated with $20 \%$ alcohol for 1 minute. Disc was carefully lifted and held at the edges by forceps vertically for few seconds to allow dripping of any extra alcohol before placement on the cornea. After ensuring that there is no more dripping the disc was carefully placed on the surface of cornea (Figure 1). Drop of fluorescein was also instilled onto the disc to track any leakage. No leak was observed during the application time.

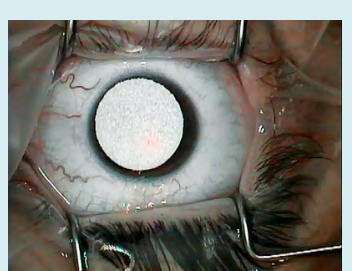

Figure 1: Alcohol impregnated Merocel disc placed on the cornea.

The disc was removed after 45 seconds and the cornea was irrigated thoroughly with copious amounts of balanced salt solution. The loose epithelium sheet was peeled in a curvilinear rhexis pattern with polyvinyl acetal sponge (Merocel; Beaver-Visitec International, Inc., Waltham, USA). No manual scraping was required and the smooth bed was exposed post epithelial removal. The bed was dried and the laser ablation was performed using the nomogram. Mitomycin-C $0.02 \%$ was applied on the ablated stroma for duration of 40 seconds. Cornea was thoroughly irrigated to wash of any residual mitomycin-C and a high water content bandage contact lens Senofilcon A (Acuvue ${ }^{\circledR}$; Johnson and Johnson Vision Care, Inc., Jacksonville, USA) was placed on the cornea and topical antibiotic and corticosteroid eye drops were instilled.

In the left eye as a control conventional alcohol well was used for epitheliectomy using $20 \%$ alcohol for duration of 20 seconds. 2 drops of fluorescein dye was instilled into the alcohol well to visualize any ooze/leakage beneath the well into the adjacent ocular surface. Mild leak through the sides of well was observed circumferentially during the application time. The loose epithelium sheet was peeled in a curvilinear rhexis pattern with polyvinyl acetal sponge (Merocel; Beaver-Visitec International, Inc., Waltham, USA). No manual scraping was required and the smooth bed was exposed post epithelial removal. The bed was dried and the laser ablation was performed using the nomogram. Mitomycin-C $0.02 \%$ was applied on the ablated stroma for duration of 40 seconds. Cornea was thoroughly irrigated to wash of any residual mitomycin-C and a high water content bandage contact lens Senofilcon A (Acuvue ${ }^{\circledR}$; Johnson and Johnson Vision Care, Inc., Jacksonville, USA) was placed on the cornea and topical antibiotic and corticosteroid eye drops were instilled. The postoperative regimen constituted $0.5 \%$ moxifloxacin hydrochloride eye drops 4 times a day (Vigamox Alcon) and prednisolone acetate 1\% ophthalmic suspension (Pred Forte Allergan) eye drops 4 times a day. The prednisolone eye drops were tapered over 4 weeks. Preservative-free Lubricants in both eyes were advised every 1 hour. 


\section{Outcome and Follow-Up}

8 eyes were studied for postoperative outcomes. In all the right eyes alcohol impregnated Merocel disc was used compared to alcohol well in the left eyes as control. Postoperative day 1 all the right eyes had better uncorrected visual acuity compared to all left eyes (Tables $1 \& 2$ ). The mean visual acuity in the right eyes on day 1 was decimal 0.95 (range 0.8 to 1.0 ) compared to mean visual acuity of 0.59 in left eyes (range 0.5. to 0.63). The pain score and inflammatory signs were much more pronounced in the left eyes as compared to the right eye. Mean Pain score was 7.5 in the left eye (range 6 to 8) as compared to 2 in the right eye (range 1 to 3 ) on first postoperative day 1 visit. The epithelial defect was same in both eyes measuring approximately 8.5 $\mathrm{mm}$. The conjunctival injection and edema ranged from severe to very severe in the left eye as compared to mild grading in the right eye (Table 1 ). The ocular surface insult was much more pronounced in the left eye as measured by the lissamine green staining.
Postoperative day 3 visit pain scores were less and vision better in both eyes. Pain scores were more in the left compared to the right eye. Mean Pain score was 5.2 in the left eye (range 5 to 6 ) as compared to 1.4 in the right eye (range 1 to 2). The mean visual acuity in the right eyes on day 3 was decimal 0.9 (range 0.8 to 1.0 ) compared to mean visual acuity of 0.8 in left eyes (range 0.63 to 1.0 ) (Tables $1 \& 2$ ). There was further improvement in conjunctival congestion and edema in both eyes. Postoperatively 1 week the visual recovery and epithelial healing was equal in both eyes with $20 / 20$ vision (decimal 1.0), without much difference in the pain between both eyes. Mean pain score in the right eye was 0.5 (range 0 to 2) and in the left eye was 1.75(range 1 to 2). Bandage contact lens was removed at this visit. Intraocular pressures were tested after 1 week on removal of bandage contact lens and they were found within normal limits in both eyes. Postoperatively 2 weeks there was no pain at all, all the patients had uncorrected 20/20 vision in both with complete healing and no signs of Conjunctiva congestion or lid oedema. Patients were followed up for next 6 months with stable visual acuity.

\begin{tabular}{|c|c|c|c|c|c|}
\hline $\begin{array}{l}\text { Number of } \\
\text { Patients }\end{array}$ & $\begin{array}{c}\text { Postoperative } \\
\text { Visit }\end{array}$ & $\begin{array}{l}\text { Subjective } \\
\text { Pain Scores }\end{array}$ & UDVA & Size of Epithelial Defect & $\begin{array}{l}\text { Conjunctival } \\
\text { Congestion and } \\
\text { Edema }\end{array}$ \\
\hline Patient 1 right eye & Day 1 & 2 out of 10 & $20 / 25$ decimal 0.8 & $8.5 \mathrm{~mm}$ & Mild \\
\hline \multirow{2}{*}{ Patient 1 left eye } & \multirow{2}{*}{ Day 1} & \multirow{2}{*}{7 out of 10} & $20 / 30$ & \multirow{2}{*}{$8.5 \mathrm{~mm}$} & \multirow{2}{*}{ Very severe } \\
\hline & & & decimal 0.63 & & \\
\hline \multirow{2}{*}{ Patient 1 right eye } & \multirow{2}{*}{ Day 3} & \multirow{2}{*}{2 out of 10} & $20 / 25$ & \multirow{2}{*}{$5 \mathrm{~mm}$} & \multirow{2}{*}{ Mild } \\
\hline & & & decimal 0.8 & & \\
\hline \multirow{2}{*}{ Patient 1 left eye } & \multirow{2}{*}{ Day 3} & \multirow{2}{*}{5 out of 10} & $20 / 25$ & \multirow{2}{*}{$5 \mathrm{~mm}$} & \multirow{2}{*}{ Severe } \\
\hline & & & decimal 0.8 & & \\
\hline Patient 1 right eye & Day 7 & 1 out of 10 & $20 / 20$ decimal 1.0 & $1 \mathrm{~mm}$ & Resolved completely \\
\hline Patient 1 left eye & Day 7 & 2 OUT OF 10 & $20 / 20$ decimal 1.0 & $1 \mathrm{~mm}$ & Mild \\
\hline Patient 1 right eye & Day 14 & 0 out of 10 & $20 / 15$ decimal 1.25 & Healed completely & Resolved completely \\
\hline Patient 1 left eye & Day 14 & 0 out of 10 & $20 / 15$ decimal 1.25 & Healed completely & Resolved completely \\
\hline Patient 2 right eye & Day 1 & 2 out of 10 & $20 / 20$ decimal 1.0 & $8.5 \mathrm{~mm}$ & Mild \\
\hline Patient 2 left eye & Day 1 & 8 out of 10 & 20/30decimal 0.63 & $8.5 \mathrm{~mm}$ & Severe \\
\hline Patient 2 right eye & Day 3 & 2 out of 10 & $20 / 20$ decimal 1.0 & $6 \mathrm{~mm}$ & Mild \\
\hline Patient 2 left eye & Day 3 & 5 out of 10 & $20 / 20$ decimal 1.0 & $5.5 \mathrm{~mm}$ & Severe \\
\hline Patient 2 right eye & Days 7 & 1 out of 10 & $20 / 20$ decimal 1.0 & $0.5 \mathrm{~mm}$ & Resolved completely \\
\hline Patient 2 left eye & Days 7 & 2 out of 10 & $20 / 20$ decimal 1.0 & Almost healed & Mild \\
\hline Patient 2 right eye & Day 14 & 0 out of 10 & $20 / 20$ decimal 1.0 & Healed completely & Resolved completely \\
\hline Patient 2 left eye & Day 14 & 0 out of 10 & 20/20 decimal 1.0 & Healed completely & Resolved completely \\
\hline
\end{tabular}


Open Access Journal of Ophthalmology

\begin{tabular}{|c|c|c|c|c|c|}
\hline Patient 3 right eye & Day 1 & 3 out of 10 & $20 / 20$ decimal 1.0 & $8 \mathrm{~mm}$ & Mild \\
\hline Patient 3 left eye & Day 1 & 7 out of 10 & 20/30decimal 0.63 & $8 \mathrm{~mm}$ & Very severe \\
\hline Patient 3 right eye & Day 3 & 1 out of 10 & $20 / 25$ decimal 0.8 & $5.5 \mathrm{~mm}$ & Mild \\
\hline Patient 3 left eye & Day 3 & 6 out of 10 & $20 / 20$ decimal 1.0 & $5 \mathrm{~mm}$ & Severe \\
\hline Patient 3 right eye & Days 7 & 0 out of 10 & $20 / 20$ decimal 1.0 & Almost healed & Resolved completely \\
\hline Patient 3 left eye & Day 7 & 1 out of 10 & $20 / 20$ decimal 1.0 & Almost healed completely & Mild \\
\hline Patient 3 right eye & Day 14 & 0 out of 10 & $20 / 20$ decimal 1.0 & Healed completely & Resolved completely \\
\hline Patient 3 left eye & Day 14 & 0 out of 10 & $20 / 20$ decimal 1.0 & Healed completely & Resolved completely \\
\hline Patient 4 right eye & Day 1 & 1 out of 10 & $20 / 20$ decimal 1.0 & $8.5 \mathrm{~mm}$ & Mild \\
\hline Patient 4 left eye & Day 1 & 8 out of 10 & $20 / 40$ decimal 0.5 & $8.5 \mathrm{~mm}$ & Very severe \\
\hline Patient 4 right eye & Day 3 & 1 out of 10 & $20 / 20$ decimal 1.0 & $5 \mathrm{~mm}$ & None \\
\hline Patient 4 left eye & Day 3 & 5 out of 10 & 20/30decimal 0.63 & $5 \mathrm{~mm}$ & Severe \\
\hline Patient 4 right eye & Days 7 & 0 out of 10 & $20 / 20$ decimal 1.0 & Almost healed completely & None \\
\hline Patient 4 left eye & Days 7 & 2 out of 10 & $20 / 20$ decimal 1.0 & $1 \mathrm{~mm}$ epithelial defect & Mild \\
\hline Patient 4 right eye & Day 14 & 0 out of 10 & $20 / 20$ decimal 1.0 & Healed completely & None \\
\hline Patient 4 left eye & Day 14 & 0 out of 10 & $20 / 20$ decimal 1.0 & Healed completely & None \\
\hline
\end{tabular}

Table 1: Post-operative outcomes of 8 eyes (4 patients).

\begin{tabular}{|c|c|c|}
\hline Post-Operative Visit & Mean Pain Score & UDVA (Uncorrected Distance Visual Acuity) \\
\hline \multirow{5}{*}{ Day 1} & 2 in the right eye (range 1 to 3 ) & 0.92 right eyes \\
\hline & & decimal(range 0.8 to 1.0 ) \\
\hline & & \\
\hline & 7.2 in left eye (range 6 to 8 ) & \\
\hline & & 0.6 left eyes (range 0.5 . to 0.63 ). \\
\hline \multirow{6}{*}{ Day 3} & 1.4 in right eye (range 1 to 2 ). & 0.88 right \\
\hline & & Eyes \\
\hline & & decimal(range 0.8 to 1.0 ) \\
\hline & 5.2 in the left eye (range 5 to 6 ) & \\
\hline & & 0.8 left eyes \\
\hline & & Decimal (range 0.63 to 1 ) \\
\hline \multirow{3}{*}{ Day 7} & 0.4 in right eye (range 0 to 2 ) & \multirow{3}{*}{ Decimal 1.0 in both right and left eyes } \\
\hline & & \\
\hline & 1.6 in left eye (range 1 to 2 ) & \\
\hline Day 14 & 0 in both right and left eyes & Decimal 1.0 in both right and left eyes \\
\hline
\end{tabular}

Table 2: Mean pain score and uncorrected distance visual acuity in right and left eyes at different time intervals $(\mathrm{p}=0.02)$.

\section{Discussion}

Photorefractive keratectomy has been used as a refractive modality of choice in thin corneas, borderline topographies with the suspicion of keratoconus in patients who are not eligible candidates for laser in situ keratomileusis (LASIK) $[1,2]$. Alcohol superseded over manual scraping as a method for epitheliectomy in photorefractive keratectomy (PRK) due 


\section{Open Access Journal of Ophthalmology}

to quicker visual rehabilitation and less incidence of haze. There is increased incidence of anterior stromal reflectivity and keratocyte density after manual scraping [3]. Alcohol epitheliectomy leaves a smooth bed with a faster healing [4]. The downside is usually there is seepage of alcohol beneath the alcohol well into the adjacent conjunctivae and ocular surface causing severe postoperative pain and inflammation. Transepithelial PRKhas a faster healing and less postoperative discomfort compared to conventional alcohol epitheliectomy using alcohol well [5-7]. The limitations of transepithelial PRK is availability of SCHWIND-ESIRIS excimer laser platform Shapira, et al. [8] have reported the long-term results of alcohol assisted epitheliectomy to be superior over mechanical debridement as well as trans- epithelial PRK [8]. We also witnessed seepage of alcohol between the alcohol well using fluorescein wherein intraoperatively fluorescein track could be recognized near the limbus trickling into the inferior fornix exposing the ocular surface to the toxic effects of alcohol. This results in severe postoperative pain and inflammation.

The novel technique of alcohol impregnated disc prevents any seepage/leakage of alcohol into the adjacent ocular surface and is as effective as using alcohol well. The only difference was that the contact time was prolonged to 45 seconds for effective loosening of epithelium. We did not use manual scraping in our case. There was no increased incidence of haze or delayed epithelial healing time because of increased contact time of cornea with alcohol. There was significant difference in the uncorrected visual acuity postoperative pain scores, lid edema, conjunctival congestion as well as first post-operative vision between both eyes leading to better patients satisfaction postoperatively. This describes an innovative technique using a simple and cost effective tool (alcohol impregnated merocel disc) for epitheliectomy. This bypasses the adverse effects of alcoholinduced postoperative inflammation and pain thereby leading to much less pain, quicker visual recovery and better patients satisfaction postoperatively. It maintains the outcome similar to transepithelial PRK without being limited by the availability of specific laser platform.

\section{Learning Points/Take Home Messages}

\section{What Was Known}

a) There is increased incidence of anterior stromal reflectivity and keratocyte density after manual scraping.

b) Alcohol epitheliectomy is superior to manual scraping and leaves a smooth bed with a faster healing.

c) Seepage of alcohol into adjacent conjunctiva causes postoperative pain and inflammation

\section{What This Paper Adds}

a) The novel technique of alcohol impregnated disc prevents any seepage/leakage of alcohol into the adjacent ocular surface.

b) Postoperative pain scores, lid edema, conjunctival congestion were much reduced and the first postoperative day vision was better compared to the eye in which alcohol well was used.

\section{References}

1. Ambrosio R, Wilson S (2003) LASIK vs LASEK vs PRK: Advantages and indications. Semin Ophthalmol 18(1): 2-10.

2. Steinert RF, Bafna S (1998) surgical correction of moderate myopia: Which method should you choose? II. PRK and LASIK are the treatments of choice. Surv Ophthalmol 43(2): 157-179.

3. Einollahi B, Rafii AB, Kanavi MR, Eslani M, Parchegani $M R$, et al. (2011) Mechanical versus alcohol-assisted epithelial debridement during photorefractive keratectomy: a confocal microscopic clinical trial. J Refract Surg 27(12): 887-893.

4. Browning AC, Shah S, Dua HS, Maharajan SV, Gray T, et al. (2003) Alcohol debridement of the corneal epithelium in PRK and LASEK: an electron microscopic study. Invest Ophthalmol Vis Sci 44(2): 510-513.

5. Naderi M, Jadidi K, Mosavi SA, Daneshi SA (2016) Transepithelial Photorefractive Keratectomy for Low to Moderate Myopia in Comparison with Conventional Photorefractive Keratectomy. J Ophthalmic Vis Res 11(4): 358-362.

6. Fattah MA, Antonios R, Arba Mosquera S, Abiad B, Awwad ST (2018) Epithelial Erosions and Refractive Results After Single-Step Transepithelial Photorefractive Keratectomy and Alcohol-Assisted Photorefractive Keratectomy in Myopic Eyes: A Comparative Evaluation Over 12 Months. Cornea 37(1): 45-52.

7. Wang DM, Du Y, Chen GS, Tang LS, He JF (2012) Transepithelial photorefractive keratectomy mode using Schwind-Esiris excimer laser: initial clinical results. Int J Ophthalmol 5(3): 334-337.

8. Shapira Y, Mimouni M, Levartovsky S, Varssano D, Sela T, et al. (2015) Comparison of Three Epithelial Removal Techniques in PRK: Mechanical, Alcohol-assisted, and Transepithelial Laser. J Refract Surg 31(11): 760-766. 